\title{
Severity of early allograft dysfunction following donation after circulatory death liver transplantation: a multicentre study
}

\author{
Kun Wang ${ }^{1,2 \#}$, Di Lu, ${ }^{1,2 \#}$, Yuhui Liu ${ }^{3 \#}$, Wangyao $\mathrm{Li}^{1,2}$, Li Zhuang ${ }^{4}$, Zhenyu Ma ${ }^{5}$, Qinfen Xie ${ }^{4}$, Binhua Pan ${ }^{1,2}$, \\ Yichao Wu ${ }^{1,2}$, Junli Chen ${ }^{6}$, Lidan Lin ${ }^{6}$, Xiaowen Feng ${ }^{1,2}$, Qiang Wei ${ }^{1,2}$, Xuyong Wei ${ }^{1,2}$, Haiyang Xie ${ }^{1,2}$, \\ Zhengxin Wang ${ }^{5}$, Shusen Zheng ${ }^{1,2,4}$, Xiao $\mathrm{Xu}^{1,2}$ \\ ${ }^{1}$ Department of Hepatobiliary and Pancreatic Surgery, First Affiliated Hospital, Zhejiang University School of Medicine, Hangzhou, China; ${ }^{2}$ Key Lab \\ of Combined Multi-Organ Transplantation, Ministry of Public Health, Hangzhou, China; ${ }^{3}$ Department of Surgery, Dezhou People's Hospital, Dezhou, \\ China; ${ }^{4}$ Department of Hepatobiliary and Pancreatic Surgery, Shulan (Hangzhou) Hospital, Hangzhou, China; ${ }^{5}$ Department of General Surgery, Huashan \\ Hospital, Fudan University, Shanghai, China; ${ }^{6}$ China Liver Transplant Registry, Hangzhou, China \\ Contributions: (I) Conception and design: X Xu; (II) Administrative support: S Zheng, Z Wang; (III) Provision of study material or patients: All authors; (IV) \\ Collection and assembly of data: All authors; (V) Data analysis and interpretation: K Wang, D Lu, Y Liu, W Li; (VI) Manuscript writing: All authors; (VII) \\ Final approval of manuscript: All authors. \\ \#These authors contributed equally to this work. \\ Correspondence to: Zhengxin Wang, MD, PhD. Professor of Surgery, Department of General Surgery, Huashan Hospital, Fudan University, Shanghai \\ 200040, China. Email: wangzhengxinhsh@163.com; Shusen Zheng, MD, PhD. Professor of Surgery, Department of Hepatobiliary and Pancreatic Surgery, \\ The First Affiliated Hospital, Zhejiang University School of Medicine, 79 Qingchun Road, Hangzhou 310003, China; Department of Hepatobiliary \\ and Pancreatic Surgery, Shulan (Hangzhou) Hospital, Hangzhou 310004, China. Email: zyzss@zju.edu.cn; Xiao Xu, MD, PhD. Professor of Surgery, \\ Department of Hepatobiliary and Pancreatic Surgery, The First Affiliated Hospital, Zhejiang University School of Medicine, 79 Qingchun Road, Hangzhou \\ 310003, China. Email: zjxu@zju.edu.cn.
}

Background: Early allograft dysfunction (EAD) is associated with decreased graft and patient survival rates. This study aimed to identify the severity of EAD and develop a predictive model for EAD after donation after circulatory death (DCD) liver transplantation (LT). Furthermore, the influence of operative time on EAD incidence was also evaluated.

Methods: In this retrospective, multicentre cohort study, nomograms were established based on a singlecentre training cohort $(n=321)$ and validated in a 3 -center validation cohort $(n=501)$.

Results: The incidence rate of EAD was 46.4\% (149/321) in the training cohort and 40.5\% (203/501) in the validation cohort. Of the $149 \mathrm{EAD}$ patients in the training cohort, 77 patients with either elevated alanine aminotransferase (ALT) or aspartate aminotransferase (AST) were classified as having EAD type A, and the rest of the EAD patients were classified as having EAD type B. Recipients with EAD type B had lower graft and patient survival rates than recipients with $\mathrm{EAD}$ type $\mathrm{A}(\mathrm{P}=0.043$ and 0.044 , respectively). We further developed a nomogram to predict EAD (graft weight, cold ischemia time, donor age, model for endstage liver disease (MELD) score) and another nomogram to predict EAD type B (graft weight, cold ischemia time, MELD score). The nomograms for the prediction of EAD and EAD type $\mathrm{B}$ had good discrimination [concordance index (C-index) =0.712 (0.666-0.758), 0.707 (0.641-0.773)] and calibration [Hosmer-Lemeshow (HL) $\mathrm{P}=0.384, \mathrm{P}=0.425$ ] in the validation cohort. An increased operative time $(>6 \mathrm{~h})$ was associated with increased $\mathrm{EAD}$ and $\mathrm{EAD}$ type $\mathrm{B}$ incidence in the high-risk group $(\mathrm{P}=0.005, \mathrm{P}=0.020$, respectively).

Conclusions: EAD type B was associated with decreased graft and patient survival rates. The novel nomograms effectively predicted the incidence of EAD and EAD type B in DCD LT patients.

Keywords: Liver transplantation; donation after circulatory death; early allograft dysfunction (EAD)

Submitted Jun 11, 2019. Accepted for publication Aug 27, 2019.

doi: $10.21037 /$ hbsn.2019.09.02

View this article at: http://dx.doi.org/10.21037/hbsn.2019.09.02 


\section{Introduction}

Liver transplantation (LT) is an established and effective treatment for patients with end-stage liver diseases; the 1 -year post-LT survival rate approximately $85 \%(1,2)$. Donation after circulatory death (DCD) is an important means to expand the donor pool to meet the growing demand for LTs (3). Early allograft dysfunction (EAD), which is associated with morbidity and mortality, is a clinical concept that describes sub-optimal liver function following LT $(4,5)$. Understanding risk factors for EAD and developing preventative and therapeutic strategies should remain a focus of research to improve both graft and patient survival. In comparison to donation after brain death (DBD) liver allografts, DCD allografts tend to have increased EAD incidence rates $(6,7)$.

Organ donation in China has increased tremendously since 2015 when voluntary donation became the only legitimate source of organ transplant. Under these circumstances, multicentre clinical studies focusing on EAD in DCD LT will be insightful and instructive in China. In this study, we analysed the incidence rates, risk factors, classification and prognosis of EAD in LT from DCD donors and successfully established a nomogram to individually predict EAD before LT surgery. Furthermore, the effect of the LT operative time on EAD incidence was also evaluated. We present the following article in accordance with the STROBE reporting checklist (available at https://hbsn.amegroups.com/article/view/10.21037/ hbsn.2019.09.02/rc).

\section{Methods}

\section{Study population}

By excluding recipients under 18 years old, re-transplant recipients, multi-organ transplant recipients, recipients with inadequate follow-up for assessing EAD and recipients missing essential data for analysis, we finally enrolled a total of 321 consecutive LT patients in the training cohort from the First Affiliated Hospital of Zhejiang University School of Medicine (Hangzhou, China) between January 2015 and June 2017. The median age of the training cohort was 49.58 (15.29) years, and $14.95 \%$ (48/321) of the recipients were female.

For the validation cohort, a total of 501 recipients were enrolled with the same criteria as the training cohort. Among the 501 recipients in the validation cohort, 209 recipients were enrolled at the First Affiliated Hospital of Zhejiang University School of Medicine (Hangzhou, China) between July 2017 and January 2019; 181 recipients were enrolled at the Shulan (Hangzhou) Hospital (Hangzhou, China) between July 2017 and December 2018, and 111 recipients were enrolled at the Huashan Hospital, Fudan University (Shanghai, China), between December 2016 and January 2018. The median age of the validation cohort was 52.17 (13.79) years, and $17.56 \%$ (88/501) of the recipients were female. The present study was conducted in accordance with the Declaration of Helsinki (as revised in 2013). This study was approved by ethical committee of each participating hospital according to the guidelines of the Regulations on Human Organ Transplantation and national legal requirements. Informed consent was taken from all individual participants. No organs from executed prisoners were used.

\section{Definition of EAD}

EAD was defined by the presence of 1 or more of the following variables: (I) total bilirubin (TB) $\geq 10 \mathrm{mg} / \mathrm{dL}$ on postoperative day 7; (II) an international normalized ratio (INR) $\geq 1.6$ on postoperative day 7 , or (III) alanine aminotransferase (ALT) or aspartate aminotransferase (AST) levels $>2,000 \mathrm{U} / \mathrm{L}$ within the first 7 postoperative days (8). Each case was classified as "EAD" or "non-EAD" according to these diagnostic criteria.

\section{Procurement of allografts from donors}

The procedure was performed in accordance with the national guidelines for DCD in China. All of the DCD donors received intravenous heparin before withdrawal of medical support. Withdrawal of the life support system mostly took place in the operating room under the supervision of senior anesthesiologists and intensive care unit (ICU) physicians. Cardiac death was declared by 3 physicians unrelated to the surgical procurement or transplant team following a 2 to 5 minutes mandatory waiting period from the time of cardiac asystole. Then, organ procurement would be initiated as soon as possible. In addition, the donor liver was perfused through abdominal aorta and the superior mesenteric vein using 4 to $8 \mathrm{~L}$ cold preservation solution.

\section{Data collection}

The following donor data were recorded for analysis: 
Table 1 Characteristics of patients in the training and validation cohort

\begin{tabular}{|c|c|c|}
\hline Characteristics & $\begin{array}{l}\text { Training cohort, } \\
\qquad \mathrm{n}=321\end{array}$ & $\begin{array}{l}\text { Validation cohort, } \\
\qquad \mathrm{n}=501\end{array}$ \\
\hline Recipient age (years) & $49.58(15.29)$ & $52.17(13.79)$ \\
\hline \multicolumn{3}{|l|}{ Recipient sex } \\
\hline Male & $273(85.05)$ & $413(82.44)$ \\
\hline Female & 48 (14.95) & $88(17.56)$ \\
\hline Recipient BMI & $22.84(3.56)$ & 22.58 (4.09) \\
\hline \multicolumn{3}{|l|}{ High MELD } \\
\hline No & $91(28.35)$ & $206(41.12)$ \\
\hline Yes & $230(71.65)$ & $295(58.88)$ \\
\hline Operative time (h) & $5.13(1.23)$ & $5.67(2.27)$ \\
\hline CIT (h) & $9.53(4.99)$ & $8.00(3.07)$ \\
\hline Donor age (years) & 41.33 (21.29) & 49.17 (18.33) \\
\hline \multicolumn{3}{|l|}{ Donor sex } \\
\hline Male & $269(83.80)$ & $406(81.04)$ \\
\hline Female & $52(16.20)$ & 95 (18.96) \\
\hline Graft weight (g) & 1,340.00 (348.00) & 1,320.00 (375.00) \\
\hline GRWR (\%) & $2.05(0.72)$ & $2.02(0.73)$ \\
\hline \multicolumn{3}{|l|}{ EAD incidence } \\
\hline EAD & $149(46.42)$ & 203 (40.52) \\
\hline Non-EAD & $172(53.58)$ & $298(59.48)$ \\
\hline
\end{tabular}

EAD, early allograft dysfunction; GRWR, graft-to-recipient weight ratio; LT, liver transplant; MELD, Model for End-stage Liver Disease; CIT, cold ischemia time. High MELD, preoperative MELD score $>30$.

age, sex, weight, height, body mass index (BMI), biopsydetermined macrovesicular steatosis, graft weight, graft weight-to-recipient ratio (GRWR) and serum sodium. The following recipient data were recorded for analysis: age, sex and model for end-stage liver disease (MELD) score prior to LT. Operative data included warm ischemic time (WIT), cold ischemic time (CIT) and operative time. WIT was defined as the interval from asystole to the beginning of cold perfusion of the allograft. CIT was defined as the interval from the beginning of cold perfusion to the removal of the allograft from cold storage.

\section{Statistical analysis}

Donor, recipient and procedural characteristics were compared between the EAD and non-EAD groups. Statistical analyses to identify prognostic factors were performed using SPSS, version 21.0 (Chicago, IL, USA). Categorical variables were expressed as numbers (percentages) and were compared using the chi-squared test. Continuous variables were presented as means \pm standard deviations (SDs) or medians and interquartile ranges (IQRs) as appropriate for the data type. The Kolmogorov-Smirnov test was used to evaluate the normality of the data distribution. Normally distributed data were compared using Student's $t$-tests, while non-normally distributed continuous variables were compared using Mann-Whitney U-tests. Variables with a $\mathrm{P}$ value $<0.05$ were subsequently entered into a multivariate analysis using a binary logistic regression method.

On the basis of the multivariate analysis, nomograms for EAD were formulated by using the rms package in $\mathrm{R}$ version 3.5.0 (http://www.r-project.org/). The discrimination of the nomogram was evaluated by the concordance index (C-index). Bootstraps with 1,000 resamples were used to validate the nomogram and construct the calibration curve. The Hosmer-Lemeshow (HL) goodness-of-fit test was used to assess the calibration of the model. The value of the C-index ranges from 0.5 to 1.0 , with 0.5 indicating a random chance and 1.0 indicating a perfect ability to correctly predict the outcome. Graft and patient survival rates were calculated using the Kaplan-Meier method and compared using the log-rank test. A P value $<0.05$ was considered statistically significant.

\section{Results}

\section{Patient characteristics}

A total of 822 LT patients were enrolled in this study. The incidence rate of EAD was $46.4 \%$ (149/321) in the training cohort, and the median follow-up time was 36.7 (11.0) months. In the validation cohort, $40.5 \%$ (203/501) of the recipients developed EAD, and the median follow-up time was 8.0 (8.3) months. Baseline characteristics for the patients are shown in Table 1 .

\section{EAD classification}

We further analysed the number of recipients based on the $\mathrm{EAD}$ diagnostic criteria in the training cohorts (Figure $1 A)$. Of the $149 \mathrm{EAD}$ recipients in the training cohort, $77(51.7 \%)$ recipients presenting only elevated ALT or AST were classified as having EAD type A, and the rest of the EAD recipients were classified as having EAD type B. 
A
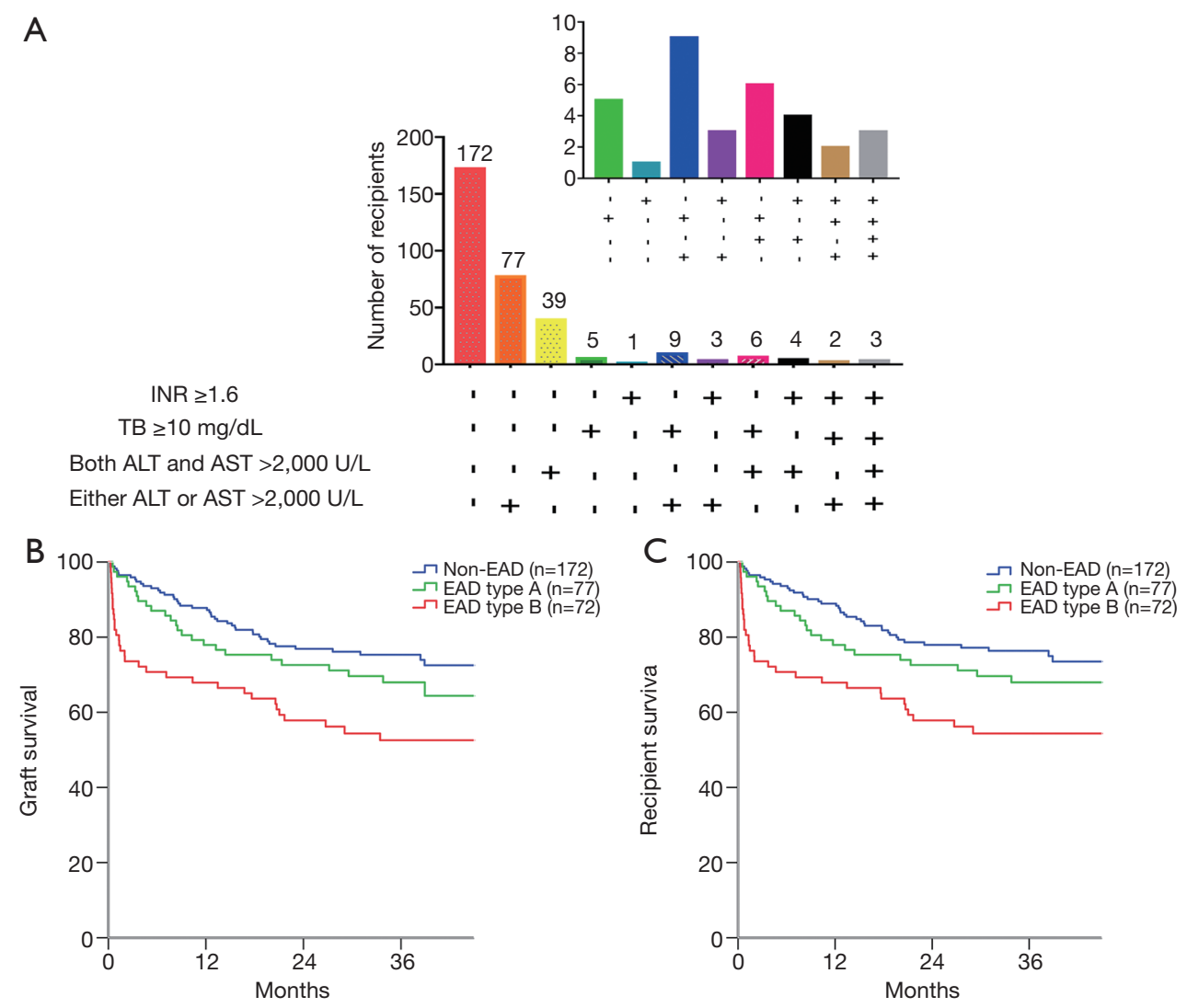

Figure 1 The proportion and Kaplan-Meier curves for recipients in the training cohort (n=321). (A) The proportion of recipients based on the EAD criteria, (B) graft survival and (C) patient survival according to the EAD type. AST/ALT >2,000 U/L: alanine aminotransferase (ALT) or aspartate aminotransferase (AST) levels $>2,000 \mathrm{U} / \mathrm{L}$ within the first 7 postoperative days: $\mathrm{TB} \geq 10 \mathrm{mg} / \mathrm{dL}$ : total bilirubin (TB) $\geq 10 \mathrm{mg} / \mathrm{dL}$ on postoperative day 7 ; INR $\geq 1.6$ : INR $\geq 1.6$ on postoperative day 7 .

Recipients with EAD type $B$ had significantly lower graft $(\mathrm{P}=0.043$, Figure $1 B)$ and patient $(\mathrm{P}=0.044$, Figure $1 C)$ survival rates than recipients with $\mathrm{EAD}$ type $\mathrm{A}$. However, graft survival $(\mathrm{P}=0.224)$ and patient survival $(\mathrm{P}=0.220)$ rates were not significantly different between the EAD type A and non-EAD recipients.

\section{Nomogram to predict EAD}

Risk factors for EAD that were identified by univariate analysis of the training cohort are presented in Table 2. Graft weight $(\mathrm{P}=0.000)$, CIT $(\mathrm{P}=0.001)$, donor age $(\mathrm{P}=0.006)$ and MELD score $(\mathrm{P}=0.048)$ were found to be independent risk factors for the incidence of $\mathrm{EAD}$ (Table 3).

To achieve the early prediction of EAD, we constructed a nomogram to predict EAD based on independent risk factors (Figure $2 A$ ). The $\mathrm{C}$-index for EAD prediction was
0.762 (95\% CI: 0.710-0.814). The HL test suggested good fitting of the model $(\mathrm{P}=0.871)$. The calibration plot showed optimal agreement between the nomogram prediction and the actual observation in the training cohort (Figure 2B).

\section{Nomogram to predict EAD type B}

Univariate and multivariate analyses of risk factors for EAD type $B$ in the training cohort are shown in Table 4. Multivariate analysis identified graft weight $(\mathrm{P}=0.000)$, CIT $(\mathrm{P}=0.018)$, and MELD score $(\mathrm{P}=0.021)$ as independent risk factors for $\mathrm{EAD}$ type $\mathrm{B}$. The nomogram to predict the individual incidence of EAD type $\mathrm{B}$ was established based on these variables (Figure 2C). The model showed good discrimination [C-index $=0.743$ (0.681-0.805)] and calibration (HL P=0.268, Figure $2 D$ ) in the training cohort. 
Table 2 Donor and recipient details for EAD and non-EAD group

\begin{tabular}{|c|c|c|c|}
\hline Characteristics & $\begin{array}{c}E A D, \\
n=149\end{array}$ & $\begin{array}{c}\text { Non-EAD, } \\
n=172\end{array}$ & $P$ value \\
\hline \multicolumn{4}{|c|}{ Donor characteristics } \\
\hline Age (years) & $42.54 \pm 12.94$ & $38.54 \pm 14.31$ & 0.009 \\
\hline Sex & & & 0.341 \\
\hline Male & $128(85.91)$ & $141(81.98)$ & \\
\hline Female & 21 (14.09) & $31(18.02)$ & \\
\hline $\mathrm{BMI}\left(\mathrm{kg} / \mathrm{m}^{2}\right)$ & $22.86(2.96)$ & $22.49(3.67)$ & 0.001 \\
\hline $\begin{array}{l}\text { Macrovesicular } \\
\text { steatosis }\end{array}$ & & & 0.031 \\
\hline$<20 \%$ & $130(87.25)$ & $162(94.19)$ & \\
\hline$\geq 20 \%$ & $19(12.75)$ & $10(5.81)$ & \\
\hline Graft weight (g) & $\begin{array}{l}1,460.00 \\
(390.00)\end{array}$ & $\begin{array}{l}1,253.00 \\
(263.00)\end{array}$ & 0.000 \\
\hline GRWR (\%) & $2.22(0.75)$ & $1.96(0.56)$ & 0.000 \\
\hline $\begin{array}{l}\text { Serum sodium } \\
(\mathrm{mmol} / \mathrm{L})\end{array}$ & $150.80(19.60)$ & $145.00(15.80)$ & 0.001 \\
\hline WIT (min) & $13.00(9.00)$ & $14.00(10.00)$ & 0.189 \\
\hline CIT (h) & $10.60(5.01)$ & $8.70(4.14)$ & 0.000 \\
\hline \multicolumn{4}{|l|}{$\begin{array}{l}\text { Recipient } \\
\text { characteristics }\end{array}$} \\
\hline Age (years) & $48.45 \pm 10.04$ & $49.67 \pm 10.49$ & 0.292 \\
\hline Sex & & & 0.474 \\
\hline Male & $129(86.58)$ & 144 (83.72) & \\
\hline Female & $20(13.42)$ & $28(16.28)$ & \\
\hline BMI $\left(\mathrm{kg} / \mathrm{m}^{2}\right)$ & $22.86(3.18)$ & $22.81(3.68)$ & 0.159 \\
\hline High MELD & & & 0.002 \\
\hline No & 94 (63.09) & $136(79.07)$ & \\
\hline Yes & 55 (36.91) & $36(20.93)$ & \\
\hline
\end{tabular}

EAD, early allograft dysfunction; BMI, body mass index; GRWR, graft-to-recipient weight ratio; LT, liver transplant; MELD, Model for End-stage Liver Disease; WIT, warm ischemia time; CIT, cold ischemia time. High MELD, preoperative MELD score $>30$.

\section{Longer operative times associated with increased EAD and EAD type B in the high-risk group}

The high risk and low risk were defined according to the predicted possibility of EAD using the established nomogram. In addition, the best cut-off value was 0.403 to classify the high and low risk group according to the
Table 3 Multivariate analysis of risk factors for EAD

\begin{tabular}{lccc}
\hline Risk factors & OR & $95 \% \mathrm{Cl}$ & $\mathrm{P}$ \\
\hline CIT & 1.146 & $1.060-1.238$ & 0.001 \\
Graft weight & 1.003 & $1.002-1.004$ & 0.000 \\
Donor age & 1.025 & $1.007-1.044$ & 0.006 \\
High MELD & 1.767 & $1.005-3.106$ & 0.048 \\
\hline OR, odds ratio; 95\% Cl, 95\% confidence interval; CIT, cold \\
ischemia time. High MELD, preoperative MELD score >30.
\end{tabular}

Youden index. According to the EAD nomogram, recipients in the training cohort were divided into a high-risk group $(\mathrm{n}=179)$ and a low-risk group $(\mathrm{n}=142)$. As shown in Table 5, an increased operative time $(>6 \mathrm{~h})$ significantly increased $\mathrm{EAD}$ and $\mathrm{EAD}$ type $\mathrm{B}$ incidence in the high-risk group $(\mathrm{P}=0.013, \mathrm{P}=0.008$, respectively). However, in the lowrisk group, $\mathrm{EAD}$ was not associated with operative time ( $\mathrm{P}=0.645, \mathrm{P}=0.327$, respectively).

\section{Validation of the results}

For the validation cohort, the number of recipients based on the EAD diagnostic criteria are shown in Figure $3 A$. Of the 203 EAD recipients in the validation cohort, 128 (63.1\%) had EAD type A. There was also a significant difference in graft survival $(\mathrm{P}=0.003$, Figure $3 B)$ and patient survival $(\mathrm{P}=0.003$, Figure $3 C)$ between $\mathrm{EAD}$ types $\mathrm{B}$ and $\mathrm{A}$ in the validation cohort. Graft survival $(\mathrm{P}=0.124)$ and patient survival $(\mathrm{P}=0.158)$ were not significantly different between $\mathrm{EAD}$ type $\mathrm{A}$ and non-EAD recipients.

In the validation cohort, the performance of the EAD nomogram was still good in terms of discrimination [C-index $=0.712(0.666-0.758)]$ and calibration $(\mathrm{HL} \mathrm{P}=0.384)$. The calibration plot showed optimal agreement between the nomogram prediction and the actual observation in the validation cohort (Figure 3D).

For the validation of the nomogram predicting EAD type $\mathrm{B}$, the model also demonstrated good discrimination, with a C-index of 0.707 (0.641-0.773), and calibration (HL $\mathrm{P}=0.425)$ in the validation group. The calibration curve demonstrated that the nomogram-predicted EAD type B incidence rate matched well with the actual observation in the validation cohort (Figure 3E).

In the validation cohort (Table 6), the increased operative time also significantly increased EAD and EAD type $\mathrm{B}$ incidence in the high-risk group $(\mathrm{P}=0.005, \mathrm{P}=0.020$, respectively). However, the incidence of EAD and $\mathrm{EAD}$ 

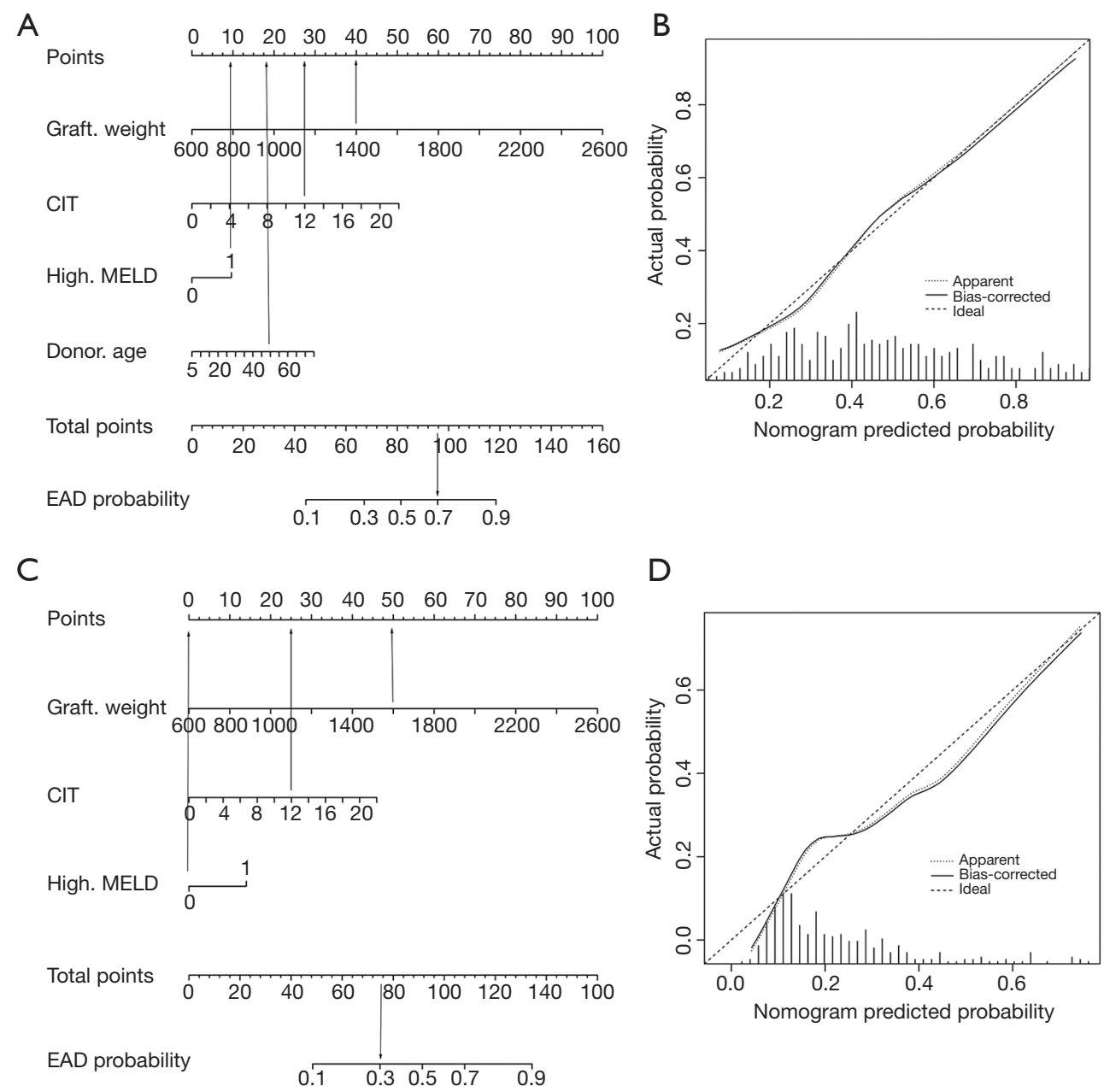

Figure 2 The nomograms to predict EAD and EAD type B. (A) the nomogram for the prediction of EAD. To use the nomogram, draw a line straight upward from the location on corresponding axis to the top line labeled "points". Sum the points for all predictors then draw a line straight downward from the axis labeled "Total Points" to find the recipient's probability of EAD. For example, in cases of a DCD liver transplant with graft weight (1,400 g), CIT (12 h), high MELD and donor age (50 years old), the points is 40, 28, 10 and 19, respectively. With the total points of 97 , the predicted probability of EAD is $70 \%$. (B) the calibration curves of the nomogram for the prediction of EAD in the internal validation. (C) the nomogram for the prediction of EAD type B. For example, in cases of a DCD liver transplant with graft weight $(1,600 \mathrm{~g})$, CIT $(12 \mathrm{~h})$, and not high MELD, the points is 50, 26 and 0, respectively. With the total points of 76, the predicted probability of EAD type B is $30 \%$. (D) the calibration curves of the nomogram for the prediction of EAD type B in the internal validation. The unit of graft weight is gram. CIT represents cold ischemia time, and the unit is hour. High MELD represents a preoperative MELD score $>30$. Nomogram-predicted probability is plotted on the $\mathrm{x}$-axis; actual probability is plotted on the $\mathrm{y}$-axis. A plot along the ideal line indicates a perfectly calibrated nomogram in which the predicted probabilities are consistent with the actual outcomes.

type B was not significantly different between relatively longer and short operative times in the low-risk group ( $\mathrm{P}=0.062, \mathrm{P}=0.053$, respectively).

\section{Discussion}

The prognostic value of EAD has been reported in many studies since it was introduced in 1987 (9). Previous studies found that DCD allografts were often associated with greater EAD incidence rates than DBD allografts $(6,7)$. A study of 199 liver transplants demonstrated that the incidence rate of $\mathrm{EAD}$ was $55.91 \%$ (10). In this study, there was also a very high incidence rate of EAD in both cohorts. The major reason may be the relatively strict definition for 
Table 4 Univariate and multivariate analysis of risk factors for EAD type B

\begin{tabular}{|c|c|c|c|}
\hline \multirow{2}{*}{ Risk factors } & \multirow{2}{*}{$\begin{array}{l}\text { Univariate } \\
\text { analysis }(P)\end{array}$} & \multicolumn{2}{|c|}{ Multivariate analysis } \\
\hline & & OR $(95 \% \mathrm{Cl})$ & $\mathrm{P}$ \\
\hline \multicolumn{4}{|l|}{ Donor characteristics } \\
\hline Age (years) & 0.414 & - & \\
\hline Sex & 0.810 & - & \\
\hline BMI $\left(\mathrm{kg} / \mathrm{m}^{2}\right)$ & 0.131 & - & \\
\hline Macrovesicular steatosis & 0.244 & - & \\
\hline Graft weight (g) & 0.000 & $\begin{array}{c}1.003 \\
(1.002-1.004)\end{array}$ & 0.000 \\
\hline GRWR (\%) & 0.000 & - & \\
\hline Serum sodium (mmol/L) & 0.267 & - & \\
\hline CIT (h) & 0.001 & $\begin{array}{c}1.113 \\
(1.019-1.215)\end{array}$ & 0.018 \\
\hline \multicolumn{4}{|l|}{ Recipient characteristics } \\
\hline Age (years) & 0.261 & - & \\
\hline Sex & 0.643 & - & \\
\hline BMI $\left(\mathrm{kg} / \mathrm{m}^{2}\right)$ & 0.431 & - & \\
\hline High MELD & 0.001 & $\begin{array}{c}2.051 \\
(1.115-3.774)\end{array}$ & 0.021 \\
\hline
\end{tabular}

EAD, early allograft dysfunction; BMI, body mass index; GRWR, graft-to-recipient weight ratio; LT, liver transplant; MELD, Model for End-stage Liver Disease; CIT, cold ischemia time. High MELD, preoperative MELD score $>30$.

DCD LT. In addition, we found that the AST/ALT criteria accounted for a large proportion of EAD in the training and validation cohorts. However, recipients satisfying the TB or INR criteria were more common in living donor liver transplantations (LDLTs) (4). Among the EAD diagnostic criteria, a large proportion of EAD recipients presented either elevated ALT or AST. Accordingly, we further divided EAD into EAD types A and B. Recipients with EAD type $B$ had significantly worse graft and patient survival rates than those with EAD type $\mathrm{A}$ in the training and validation cohorts. There was no significant difference in graft and patient survival rates between EAD type A and non-EAD patients in either the training or validation cohorts. This classification enhances our understanding of EAD prognoses.

A large proportion of recipients develop EAD after DCD LT. It is necessary to develop a clinically useful nomogram to predict EAD incidence. For the construction of the nomogram, the training cohort included 321 recipients; this was one of the largest series to analyse the risk factors for EAD after LT with allografts from DCD donors. We identified that CIT, graft weight, donor age and MELD scores were significantly associated with EAD. We also found that graft weight, CIT, and MELD scores were independent risk factors for EAD type B.

A number of factors, including recipient-, surgical- and donor-related factors, can affect the incidence of EAD. Recipient-related factors, such as the pre-LT MELD score, could affect the occurrence of EAD. A study of 199 LT recipients found that the pre-LT MELD score was an independent risk factor for EAD (10). In the present study, we found that high MELD scores $(>30)$ were significantly associated with the incidence of EAD. Surgical-related factors such as CIT were also associated with EAD. It has been previously reported that CIT is significantly related to the incidence of EAD. Sibulesky et al. demonstrated that $53.1 \%$ of recipients in the CIT $>12 \mathrm{~h}$ group developed EAD, while only $18 \%$ in the CIT $<8$ h group developed EAD (11). CIT was associated with ischemia reperfusion injury, which was responsible for the increase in the occurrence of EAD.

Donor-related factors are composed of donor age, graft weight, donor sodium levels and graft steatosis. A study from the Mayo Clinic reported that donor age was an independent predictive factor for EAD (12). Aged donors exhibit declined hepatic progenitor cell populations and impaired liver regeneration (13). Similar results were observed in this study; donor age was an independent risk factor for the incidence of EAD. In LDLT, a GRWR $<0.8 \%$ was considered a small failure to meet the metabolic demand of the recipients. In this study, in the DCD LT cohort, the minimal GRWR was greater than $0.8 \%$, and the GRWR was not an independent risk factor for the incidence of EAD. Pomposelli et al. demonstrated that decreased graft weight in the left lobe is a risk factor for EAD in LDLT (14). We also found that recipients in the EAD group had increased graft weights. A large graft may cause relative hypoperfusion, resulting in graft injury after LT.

A study from the University of Barcelona reported that an increased donor serum sodium concentration prior to organ procurement was an independent predictive factor for early postoperative graft dysfunction after LT (15). These results were confirmed by Totsuka et al., who suggested that a final donor serum sodium beyond $155 \mathrm{mmol} / \mathrm{L}$ was strongly associated with graft loss (16). Nevertheless, a recent study including 474 cases of lung transplantation 
Table 5 The influence of longer operative time on $\mathrm{EAD}$ and $\mathrm{EAD}$ type $\mathrm{B}$ in the training cohort

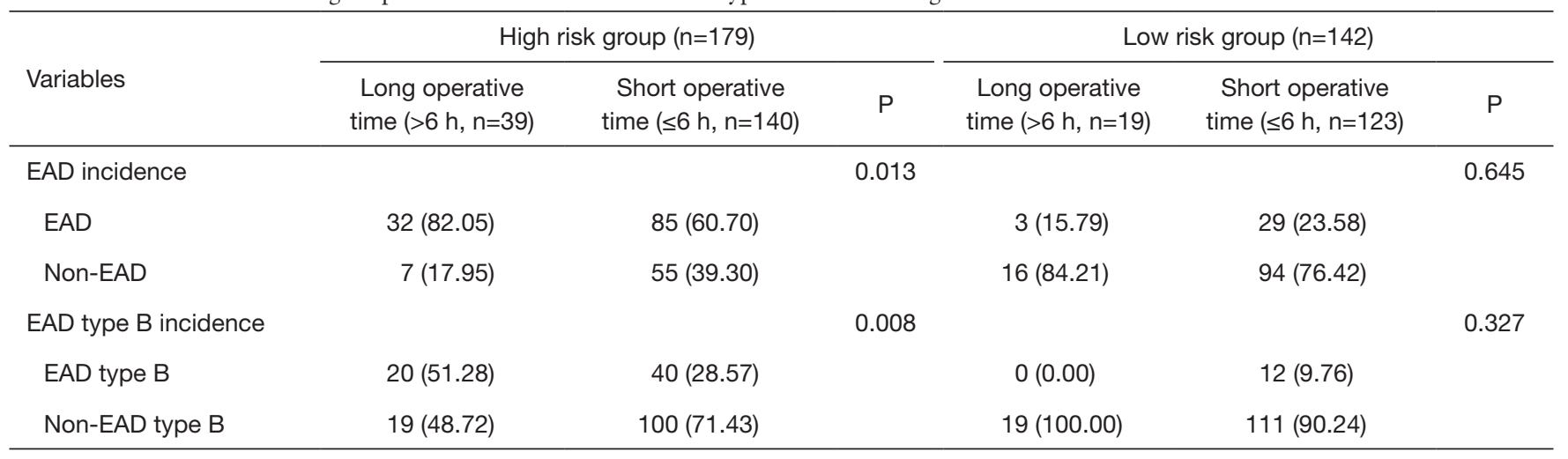

EAD, early allograft dysfunction.

found that donor hypernatraemia was not associated with primary graft dysfunction (17). Our study also found that the donor sodium concentration was not an independent risk factor for EAD after LT. Graft steatosis had a significant impact on graft survival after LT. Ali et al. divided recipients into nil $(\mathrm{n}=182)$, mild $(\mathrm{n}=186)$ and moderate $(\mathrm{n}=37)$ steatosis groups, and the incidence of EAD was $13.2 \%, 22.6 \%$ and $62.2 \%(\mathrm{P}<0.001)$, respectively $(18)$. However, our previous study found that the incidence of EAD was $56.3 \%$ in the $\geq 20 \%$ macrovesicular steatosis group and $36.0 \%$ in the $<20 \%$ macrovesicular steatosis group $(\mathrm{P}=0.12)(19)$. In the present study, we demonstrated that $\geq 20 \%$ graft macrovesicular steatosis tended to be higher in the EAD group, but it was not an independent risk factor for EAD.

Based on the four significant risk factors identified in the present study, we constructed a nomogram to predict EAD. We also developed a clinically useful nomogram to predict EAD type $\mathrm{B}$, which represents a more severe subtype of EAD and is associated with a relatively poor prognosis. The models showed good performance in discrimination and calibration in the training and validation cohorts. In addition, the C-index in the validation group was lower than those in the training group, which may due to the slightly different incidence of EAD and EAD type B in these two groups. The training group was used to establish the predictive nomogram from early era. In contrast, the validation group was used to validate the nomogram independently from recent era. In fact, the era effect in $\mathrm{EAD}$ and EAD type $\mathrm{B}$ incidence may exist in the training and validation group owing to the study design. However, the predictive nomograms are still reliable in the external validation group.

In 2015, Hoyer et al. established a formula to predict
EAD based on significant donor-related factors, the C-index, which was 0.68 and 0.622 , respectively, in the analysis cohort and validation cohort (20). Nevertheless, in their study, all recipients received liver allografts from DBD donors. Yang et al. also constructed a nomogram to predict the incidence of EAD after LT (10). However, the nomogram was derived from a relatively small sample size from a single centre and from primarily DBD donors. DCD liver allograft recipients are at a higher risk of developing EAD $(6,7)$. Therefore, we constructed this nomogram to predict $\mathrm{EAD}$ in patients with allografts obtained from DCD donors. The nomogram enables physicians to objectively identify patients at high risk of developing EAD and optimize the allocation strategy.

In the present study, we also found that an increased operative time $(>6 \mathrm{~h})$ was associated with increased EAD and EAD type $\mathrm{B}$ incidence in the high-risk group. Operative time has been considered a risk factor for outcomes. Lee et al. found that operative time significantly influenced graft survival in LT recipients (21). They also demonstrated that operative time was an independent risk factor for EAD (12). Similar results were also found in this study. An increased operative time increases the incidence of postoperative complications. To decrease the incidence rates of EAD and EAD type B in DCD LT recipients, it is necessary to control operative times, especially in the high-risk group. In addition to controlling operative time, liver protective strategies, such as $\mathrm{N}$-acetylcysteine, are also promising for preventing $\mathrm{EAD}$ if the EAD nomogram score is high $(22,23)$.

The main limitations concerning the evaluation of our data are summarized as follows. First, this was a retrospective study, and further prospective, multicentre research is required to validate the observations in this 
A

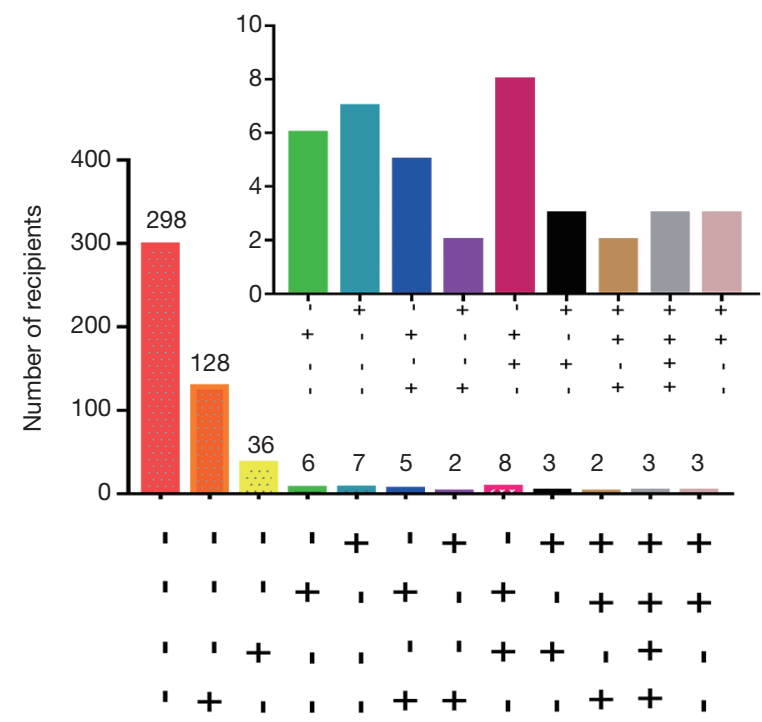

B

INR $\geq 1.6$

$\mathrm{TB} \geq 10 \mathrm{mg} / \mathrm{dL}$

Both ALT and AST >2,000 U/L

Either ALT or AST >2,000 U/L
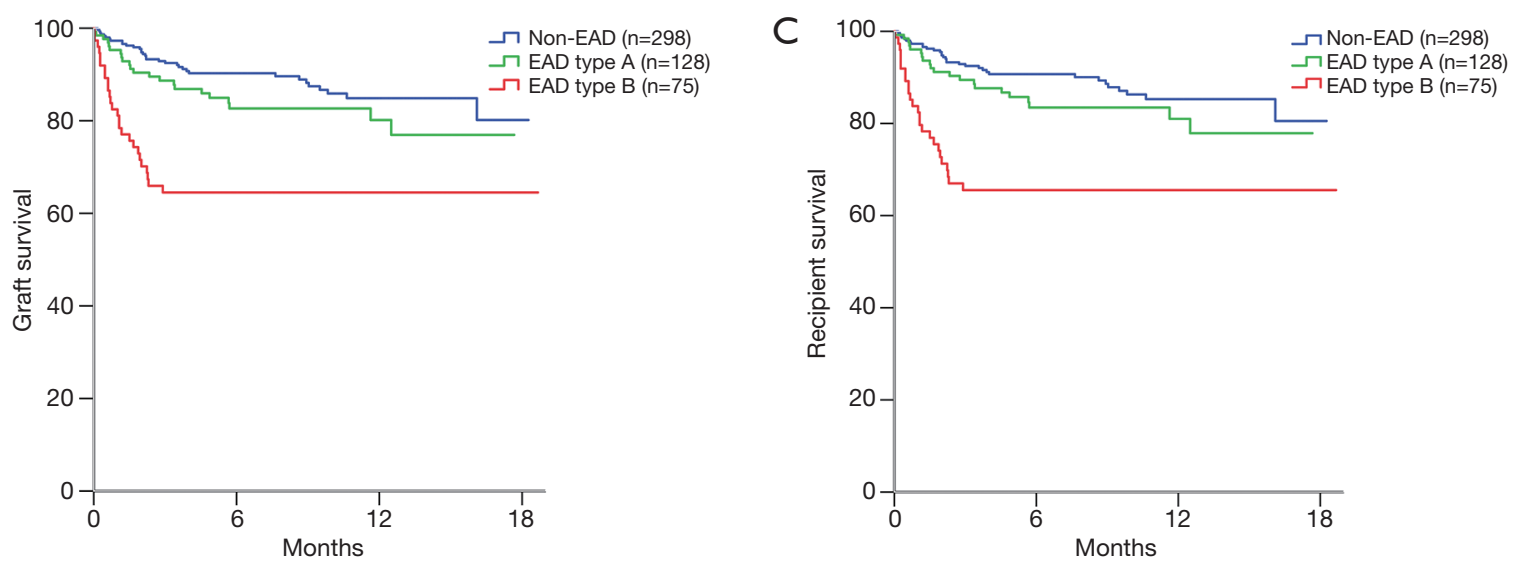

D
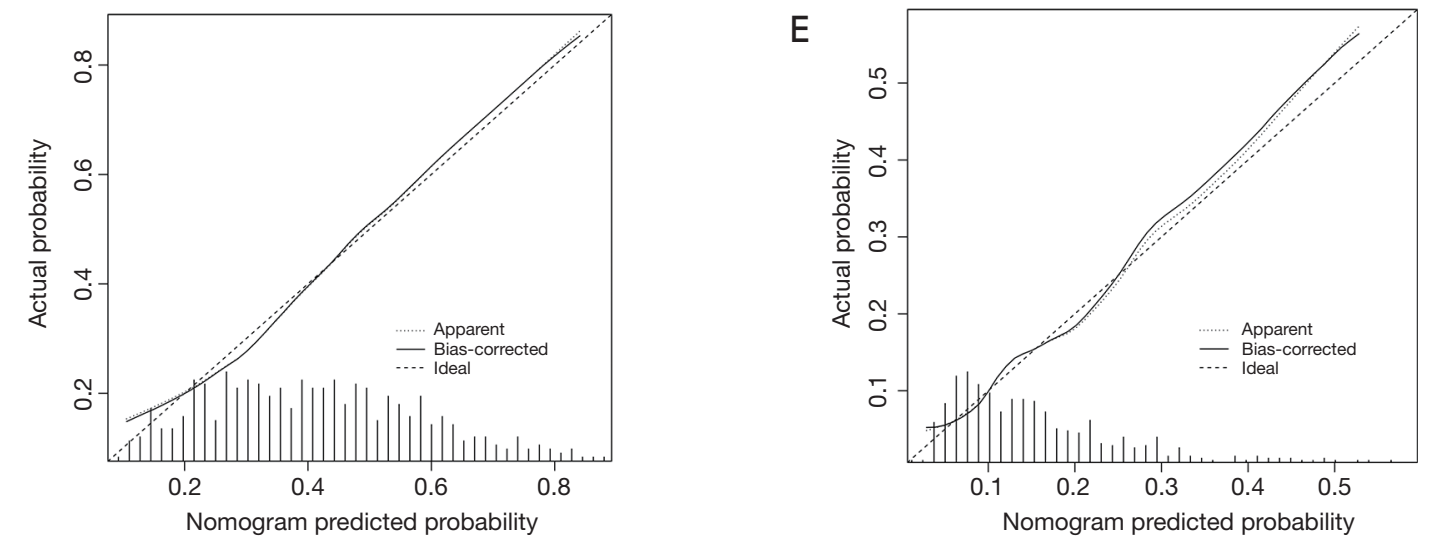

Figure 3 The proportions, Kaplan-Meier curves and calibration plots for the recipients in the validation cohort ( $\mathrm{n}=501)$. (A) The proportion of recipients based on the EAD criteria, (B) graft survival and (C) patient survival rates according to the EAD type, (D) calibration plots of the nomogram for the prediction of EAD in the external validation, (E) calibration plots of the nomogram for the prediction of EAD type $\mathrm{B}$ in the external validation. AST/ALT >2,000 U/L: alanine aminotransferase (ALT) or aspartate aminotransferase (AST) levels $>2,000 \mathrm{U} / \mathrm{L}$ within the first 7 postoperative days: $\mathrm{TB} \geq 10 \mathrm{mg} / \mathrm{dL}$ : total bilirubin (TB) $\geq 10 \mathrm{mg} / \mathrm{dL}$ on postoperative day 7; INR $\geq 1.6$ : INR $\geq 1.6$ on postoperative day 7. Nomogram-predicted probability is plotted on the $\mathrm{x}$-axis; actual probability is plotted on the $y$-axis. A plot along the ideal line indicates a perfectly calibrated nomogram in which the predicted probabilities are consistent with the actual outcomes. 
Table 6 The influence of longer operative time on EAD and EAD type B in the validation cohort

\begin{tabular}{|c|c|c|c|c|c|c|}
\hline Variables & \multicolumn{3}{|c|}{ High risk group $(n=297)$} & \multicolumn{3}{|c|}{ Low risk group $(\mathrm{n}=204)$} \\
\hline EAD incidence & & & 0.005 & & & 0.062 \\
\hline EAD & 68 (62.96) & $87(46.03)$ & & $28(29.47)$ & 20 (18.35) & \\
\hline EAD type B & $30(27.78)$ & $31(16.40)$ & & $10(10.53)$ & $4(3.67)$ & \\
\hline Non-EAD type B & 78 (72.22) & $158(83.60)$ & & $85(89.47)$ & $105(96.33)$ & \\
\hline
\end{tabular}

$E A D$, early allograft dysfunction

study. Second, the findings in our study were obtained from only adult patients who received DCD allografts; therefore, they are not applicable to paediatric recipients or adult patients who received DBD allografts.

In conclusion, recipients presenting EAD type B had lower graft and patient survival rates. The classification system facilitated the prognostic evaluation and clinical management of EAD. Moreover, the novel nomograms could effectively predict the incidence of EAD and EAD type $B$, enabling transplant physicians to objectively evaluate the risk of EAD and EAD type $\mathrm{B}$.

\section{Acknowledgments}

Funding: This study was supported by grants from the National Science and Technology Major Project (Grant number: 2017ZX10203205), National Natural Science Funds for Distinguished Young Scholar of China (Grant number: 81625003), Zhejiang Provincial Natural Science Foundation of China (Grant No. LQ17H160006) and National Natural Science Foundation of China (Grant number. 81570589,81800578$)$. The authors wish to thank the China Liver Transplant Registry (CLTR) for the data collection and statistical analysis.

\section{Footnote}

Reporting Checklist: The authors have completed the STROBE reporting checklist. Available at https://hbsn. amegroups.com/article/view/10.21037/hbsn.2019.09.02/rc

Data Sharing Statement: Available at https://hbsn. amegroups.com/article/view/10.21037/hbsn.2019.09.02/dss
Conflicts of Interest: All authors have completed the ICMJE uniform disclosure form (available at https://hbsn. amegroups.com/article/view/10.21037/hbsn.2019.09.02/coif). The authors have no conflicts of interest to declare.

Etbical Statement: The authors are accountable for all aspects of the work in ensuring that questions related to the accuracy or integrity of any part of the work are appropriately investigated and resolved. The present study was conducted in accordance with the Declaration of Helsinki (as revised in 2013). This study was approved by ethical committee of each participating hospital according to the guidelines of the Regulations on Human Organ Transplantation and national legal requirements. Informed consent was taken from all individual participants. No organs from executed prisoners were used.

Open Access Statement: This is an Open Access article distributed in accordance with the Creative Commons Attribution-NonCommercial-NoDerivs 4.0 International License (CC BY-NC-ND 4.0), which permits the noncommercial replication and distribution of the article with the strict proviso that no changes or edits are made and the original work is properly cited (including links to both the formal publication through the relevant DOI and the license). See: https://creativecommons.org/licenses/by-nc-nd/4.0/.

\section{References}

1. Lee BP, Mehta N, Platt L, et al. Outcomes of early liver transplantation for patients with severe alcoholic hepatitis. Gastroenterology 2018;155:422-30.e1.

2. Orman ES, Mayorga ME, Wheeler SB, et al. Declining 
liver graft quality threatens the future of liver transplantation in the United States. Liver Transpl 2015;21:1040-50.

3. Kollmann D, Sapisochin G, Goldaracena N, et al. Expanding the donor pool: Donation after circulatory death and living liver donation do not compromise the results of liver transplantation. Liver Transpl 2018;24:779-89.

4. Okamura Y, Yagi S, Sato T, et al. Coexistence of Bilirubin $\geq 10 \mathrm{mg} / \mathrm{dL}$ and Prothrombin Time-International Normalized Ratio $\geq 1$.6 on Day 7: A Strong Predictor of Early Graft Loss After Living Donor Liver Transplantation. Transplantation 2018;102:440-7.

5. Golse N, Guglielmo N, El MA, et al. Arterial lactate concentration at the end of liver transplantation is an early predictor of primary graft dysfunction. Ann Surg 2019;270:131-8.

6. Blasi A, Beltran J, Martin N, et al. Transient hyperglycemia during liver transplantation does not affect the early graft function. Ann Hepatol 2015;14:675-87.

7. Croome KP, Wall W, Quan D, et al. Evaluation of the updated definition of early allograft dysfunction in donation after brain death and donation after cardiac death liver allografts. Hepatobiliary Pancreat Dis Int 2012;11:372-6.

8. Olthoff KM, Kulik L, Samstein B, et al. Validation of a current definition of early allograft dysfunction in liver transplant recipients and analysis of risk factors. Liver Transpl 2010;16:943-9.

9. Makowka L, Gordon RD, Todo S, et al. Analysis of donor criteria for the prediction of outcome in clinical liver transplantation. Transplant Proc 1987;19:2378-82.

10. Yang L, Xin EY, Liao B, et al. Development and validation of a nomogram for predicting incidence of early allograft dysfunction following liver transplantation. Transplant Proc 2017;49:1357-63.

11. Sibulesky L, Li M, Hansen RN, et al. Impact of cold ischemia time on outcomes of liver transplantation: A single center experience. Ann Transplant 2016;21:145-51.

12. Lee DD, Croome KP, Shalev JA, et al. Early allograft dysfunction after liver transplantation: An intermediate outcome measure for targeted improvements. Ann Hepatol 2016;15:53-60.

13. Ono Y, Kawachi S, Hayashida T, et al. The influence of donor age on liver regeneration and hepatic progenitor cell populations. Surgery 2011;150:154-61.
14. Pomposelli JJ, Goodrich NP, Emond JC, et al. Patterns of early allograft dysfunction in adult live donor liver transplantation: The A2ALL experience. Transplantation 2016;100:1490-9.

15. Gonzalez FX, Rimola A, Grande L, et al. Predictive factors of early postoperative graft function in human liver transplantation. Hepatology 1994;20:565-73.

16. Totsuka E, Dodson F, Urakami A, et al. Influence of high donor serum sodium levels on early postoperative graft function in human liver transplantation: Effect of correction of donor hypernatremia. Liver Transpl Surg 1999;5:421-8.

17. Oude LA, Hessels L, de Vries AJ, et al. Donor hypernatremia is not related with the duration of postoperative mechanical ventilation, primary graft dysfunction, or Long-Term outcome following lung transplantation. Ann Transplant 2018;23:500-6.

18. Ali JM, Davies SE, Brais RJ, et al. Analysis of ischemia/ reperfusion injury in time-zero biopsies predicts liver allograft outcomes. Liver Transpl 2015;21:487-99.

19. Xia W, Ke Q, Wang Y, et al. Donation after cardiac death liver transplantation: Graft quality evaluation based on pretransplant liver biopsy. Liver Transpl 2015;21:838-46.

20. Hoyer DP, Paul A, Gallinat A, et al. Donor information based prediction of early allograft dysfunction and outcome in liver transplantation. Liver Int 2015;35:156-63.

21. Lee DD, Li J, Wang G, et al. Looking inward: The impact of operative time on graft survival after liver transplantation. Surgery 2017;162:937-49.

22. Goldaracena N, Echeverri J, Spetzler VN, et al. Antiinflammatory signaling during ex vivo liver perfusion improves the preservation of pig liver grafts before transplantation. Liver Transpl 2016;22:1573-83.

23. O'Leary JG, Levitsky J, Wong F, et al. Protecting the kidney in liver transplant candidates: PracticeBased recommendations from the american society of transplantation liver and intestine community of practice. Am J Transplant 2016;16:2516-31.

Cite this article as: Wang $\mathrm{K}, \mathrm{Lu} \mathrm{D}$, Liu Y, Li W, Zhuang L, Ma Z, Xie Q, Pan B, Wu Y, Chen J, Lin L, Feng X, Wei Q, Wei X, Xie H, Wang Z, Zheng S, Xu X. Severity of early allograft dysfunction following donation after circulatory death liver transplantation: a multicentre study. HepatoBiliary Surg Nutr 2021;10(1):9-19. doi: 10.21037/hbsn.2019.09.02 